\title{
Experiencia clínica en tiroidectomía total
}

\author{
Clinical experience in total thyroidectomy
}

Pablo Ortega $\mathrm{R}^{\mathbf{1}}$, Alexis Urra $\mathrm{B}^{\mathbf{1}, 2}$, Álvaro Compan J².

\begin{abstract}
RESUMEN
Introducción: La tiroidectomía total es una de las cirugías más frecuentes en cabeza y cuello. La experiencia en servicios de ORL es escasa en Chile.

Objetivo: Analizar la experiencia clínica en tiroidectomía total en el Servicio de ORL del Hospital Guillermo Grant Benavente.

Material y método: Estudio descriptivo prospectivo. Evaluación de los aspectos clínicos, la técnica quirúrgica y el porcentaje de complicaciones de los pacientes sometidos a tiroidectomía total durante el año 2009.

Resultados: Se realizaron 34 tiroidectomías totales. La indicación más frecuente fue bocio multinodular (82,3\%). Los diagnósticos más frecuentes fueron hiperplasia folicular (44\%) y carcinoma papilar (32\%). Se presentó lesión del nervio laríngeo recurrente transitoria en $8,8 \%$, no se presentaron lesiones permanentes. Se presentó hipocalcemia transitoria en $29 \%$ y permanente en sólo un paciente $(2,9 \%)$.

Conclusiones: Nuestra experiencia presenta resultados en relación a indicaciones, diagnósticos y complicaciones concordantes con los estándares aceptados por la literatura mundial.
\end{abstract}

Palabras clave: Tiroidectomía, total, complicaciones.

\begin{abstract}
Introduction: Total thyroidectomy is one of the most frequent surgeries in head and neck. Experience in ENT services is scarce in Chile.

Aim: To analyze clinical experience in total thyroidectomy in the ENT service of Guillermo Grant Benavente Hospital.

Material and method: Prospective descriptive study. Evaluation of clinical aspects, surgical technique and percentage of complications of patients submitted to total thyroidectomy during the year 2009.

Results: There were done 34 total thyroidectomies. The most frequent indication was multinodular goiter. The most frequent diagnoses were follicular hyperplasia (44\%) and papillary carcinoma (32\%). Transitory injury to the recurrent laryngeal nerve was present in $8,8 \%$. Permanent injuries did not happen. Transitory hypocalcemia was present in $29 \%$ and permanent in only one patient $(2,9 \%)$.

Conclusions: Our experience presents results in relation to indications, diagnosis and complications, in accordance with the standards of the world literature.

Key words: Thyroidectomy, Total, Complications.
\end{abstract}

\footnotetext{
${ }^{1}$ Médico Otorrinolaringólogo, Hospital Guillermo Grant Benavente.

${ }^{2}$ Médico Cirujano de Cabeza y Cuello, Hospital Guillermo Grant Benavente.
} 


\section{INTRODUCCIÓN}

La tiroidectomía es una de las cirugías más frecuentes en el campo de la cirugía de cabeza y cuello. En muchos centros alrededor del mundo es realizada por otorrinolaringólogos (ORL), pero en Chile existen escasos reportes de experiencia de ORL en tiroidectomías².

La tiroidectomía cuando es realizada por equipos especializados es una cirugía segura con mínima morbilidad y prácticamente nula mortalidad ${ }^{3}$. Es un procedimiento diagnóstico y terapéutico, que puede ser total, cuando se extirpa la glándula completa, o parcial, que dependiendo de la extensión de la resección glandular; incluye hemitiroidectomías y tiroidectomías subtotales ${ }^{1,2,4}$. Las indicaciones más frecuentes de la tiroidectomía son los nódulos tiroideos, el bocio multinodular y el cáncer de tiroides ${ }^{2-4}$.

La tiroidectomía total (TT) es la tiroidectomía más frecuente en muchos centros especializados ${ }^{5}$, debido a que en manos de cirujanos expertos no presenta mayor tasa de complicaciones que las tiroidectomías parciales ${ }^{6}$, pero sí una menor frecuencia de recidivas, permitiendo realizar seguimientos con tiroglobulina y terapias complementarias con iodo radioactivo ${ }^{7}$.

En general las complicaciones son directamente proporcionales a la extensión de la cirugía e inversamente proporcionales a la experiencia del equipo de cirujanos ${ }^{1,3}$. Las más frecuentes son hipoparatiroidismo, lesión del nervio laríngeo recurrente (NLR) y hematomas cervicales ${ }^{1,6}$. La frecuencia de ellas puede aumentar en reoperaciones y al asociar disección cervical ganglionar del nivel VI o compartimiento central ${ }^{5,8}$.

\section{OBJETIVO}

Nuestro objetivo es analizar los aspectos clínicos, la técnica quirúrgica y el porcentaje de complicaciones de los pacientes sometidos a tiroidectomía total en el Hospital Guillermo Grant Benavente.

\section{MATERIAL Y MÉTODO}

Estudio descriptivo prospectivo. Se incluyeron en el estudio todos los pacientes sometidos a tiroidectomía total durante el 1 enero hasta el 31 de diciembre de 2009. Se excluyeron aquellos pacientes sometidos a tiroidectomía parcial, las reintervenciones y las tiroidectomías que asociaron disección ganglionar cervical. Al ingreso para la cirugía se revisó la ficha clínica de cada paciente, registrando datos epidemiológicos, del estudio clínico y además se realizó una nasofibroscopía. Durante la hospitalización se registraron datos del protocolo quirúrgico, de la evolución clínica posoperatoria, una nasofibroscopía posoperatoria y calcemias cada 12 horas hasta el alta. Se definió como lesión del NLR a toda alteración de la motilidad cordal que apareciera en el posoperatorio. Se definió como hipocalcemia como un calcio menor a $8 \mathrm{gr} / \mathrm{dl}$. Al control ambulatorio a las 3 semanas se registró una calcemia y el diagnóstico de anatomía patológica. Se definió a las complicaciones como transitorias si remitían dentro de los primeros 6 meses posoperatorios y como persistentes si permanecían más allá de ese período. Los pacientes que presentaron alguna complicación fueron seguidos mensualmente. Todos los datos fueron registrados en una tabla Excel.

\section{RESULTADOS}

Durante el año 2009 se realizaron 48 tiroidectomías. De ellas 34 fueron tiroidectomías totales que cumplieron los requisitos para incluirlas en el estudio. El rango de edad fue de 17 a 76 años con un promedio de 50,4 años. El $94,1 \%$ de nuestros pacientes fueron de sexo femenino.

Todos los pacientes fueron operados por el equipo de autores. La técnica quirúrgica en todos los casos fue incisión cervical tipo Kocher, sección de músculos pretiroideos, disección capsular de glándula tiroides, sección de pedículos vasculares previa identificación del NLR y de las glándulas paratiroides (más de 2 en todos los casos), hemostasia con ligaduras, bipolar y Harmonic Scalpel Focus ${ }^{\circledR}$, sutura de músculos y drenaje aspirativo subcutáneo. La duración promedio de la TT fue de 156 minutos.

De los 34 pacientes, 28 (82,3\%) fueron derivados por bocio multinodular y 6 por bocio uninodular. El $38 \%$ $(n=13)$ de los casos presentaban un bocio con nódulos de más de 3 centímetros de diámetro a la ecografía cervical. En 24 pacientes había antecedente de punción con aguja fina (PAF) preoperatoria, el diagnóstico más frecuente fue lesión benigna en el 54,3\% (Tabla 1).

Los diagnósticos por histopatología más frecuentes fueron la hiperplasia folicular $(44,1 \%)$ y el carcino- 
Tabla 1. Diagnóstico PAF preoperatoria

\begin{tabular}{|lrr|}
\hline Diagnóstico PAF & $\mathrm{n}$ & $\%$ \\
\hline Lesión benigna & 13 & 54,2 \\
Carcinoma papilar & 6 & 25 \\
Neoplasia folicular & 5 & 20,8 \\
Total & 24 & 100 \\
\hline
\end{tabular}

Tabla 2. Diagnósticos por anatomía patológica

\begin{tabular}{|lrr|}
\hline Diagnóstico & $\mathrm{n}$ & $\%$ \\
\hline Hiperplasia folicular & 15 & 44,1 \\
Carcinoma papilar & 9 & 26,4 \\
Carcinoma papilar variante folicular & 2 & 5,9 \\
Carcinoma folicular & 2 & 5,9 \\
Adenoma folicular & 4 & 11,8 \\
Tiroiditis de Hashimoto & 2 & 5,9 \\
Total & 34 & 100 \\
\hline
\end{tabular}

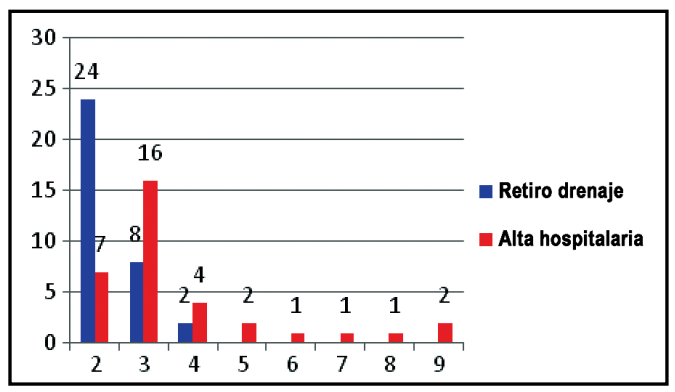

Figura 1. Número días posoperatorios y pacientes según retiro drenaje y alta hospitalaria.

ma papilar (32,3\%). El 38,2\% de los casos correspondieron a cáncer de tiroides (Tabla 2). En los pacientes que presentaban una PAF previa su correlación con el diagnóstico definitivo fue de 70,8\%. En los casos en que el diagnóstico por PAF fue carcinoma papilar la correlación fue 100\%. Ningún caso presentó complicaciones intraoperatorias. El drenaje aspirativo se retiro el día 2 en el 70,5\% ( $n=24)$ y el alta se produjo entre el 2 y 4 día en el 79,4\% ( $n=27$ ) de los casos (Figura 1). No se registraron hematomas ni seromas.

La evaluación de la motilidad cordal preoperatoria con nasofibroscopía (NFC) fue normal en todos los pacientes. En el control posoperatorio 3 pacientes $(8,8 \%)$ presentaron una alteración de la motilidad cordal unilateral concordante con lesión del NLR. Todos fueron derivados a terapia fonoaudiológica y controles mensuales con NFC objetivando la recuperación completa durante los 4 meses posteriores a la cirugía. No se registraron lesiones del NLR permanentes.

Todos los pacientes recibieron aporte preventivo de calcio oral desde el primer día posoperatorio. En la primera calcemia posoperatoria 10 pacientes $(29,4 \%)$ presentaron hipocalcemia la que en todos los casos fue asintomática. En 5 casos la estabilización de la calcemia prolongó la hospitalización permitiendo el alta entre el sexto y noveno día posoperatorio (Figura 1).

En el control ambulatorio, sólo 2 pacientes persistieron con hipocalcemia. Uno de ellos mejoró a los 4 meses y el otro completa un seguimiento de 15 meses con hipoparatiroidismo permanente $(2,9 \%)$.

\section{DISCUSIÓN}

La TT es un procedimiento seguro cuando es realizado por equipos con experiencia ${ }^{1,7}$. En el mundo es realizada por $\mathrm{ORL}$, cirujanos de cabeza y cuello y cirujanos endocrinos, pero en Chile los reportes de $\mathrm{ORL}$ son escasos ${ }^{1}$. Nuestra experiencia en $\mathrm{TT}$ es la serie más grande publicada por ORL en Chile.

En el pasado la TT ha sido reservada para el tratamiento de la patología maligna por poner en riesgo a ambos NLR y todas las paratiroides ${ }^{5}$. Pero diferentes estudios no demuestran una mayor frecuencia de complicaciones permanentes en el manejo de patología benigna al compararla con tiroidectomías parciales ${ }^{6,7}$. En nuestra casuística el $44,1 \%$ de los casos correspondieron a bocio multinodular bilateral, el que puede ser manejado en forma eficaz y segura con TT disminuyendo la frecuencia de recidivas ${ }^{7}$.

En relación a la técnica quirúrgica, utilizamos la sección de los músculos pretiroideos porque permite una mejor exposición y no aumenta la morbilidad. Además ocupamos la Harmonic Scalpel Focus ${ }^{\circledR}$ para la hemostasia, que ha demostrado en la literatura disminuir el tiempo quirúrgico sin aumentar las complicaciones ${ }^{9-11}$.

El uso de drenajes es controvertido en cirugía tiroidea $^{12}$. Nosotros utilizamos un drenaje aspirativo subcutáneo, para evitar un contacto directo del drenaje con las paratiroides y el NLR. En nuestra experiencia es efectivo, seguro y no presentó complicaciones asociadas. 
Las complicaciones de la $\Pi$ más temidas son la lesión del NLR y la hipocalcemia ${ }^{13}$. La frecuencia esperada de hipocalcemia posoperatoria es hasta $40 \%$. La gran mayoría de las hipocalcemias $7 \%$, con lesiones permanentes idealmente no mayores al $1 \%{ }^{1,17,18}$. El monitoreo del nervio recurrente no ha demostrado disminuir la frecuencia de esta complicación ${ }^{17}$. En nuestra serie registramos un caso $(2,9 \%)$ de hipocalcemia permanente y ningún caso de lesión NLR permanente.

El uso de calcio oral preventivo en el posoperatorio ha demostrado disminuir la frecuencia de hipocalcemias sintomáticas ${ }^{14}$. En nuestra serie todos recibieron aporte preventivo de calcio y ninguna de las hipocalcemias desarrollo síntomas.

\section{CONCLUSIÓN}

La $\Pi$ es una cirugía segura cuando es realizada por equipos experimentados. Puede ser indicada para manejo de patología maligna como benigna, sin mayores complicaciones que las cirugías parciales. Nuestra experiencia presenta resultados en relación a indicaciones, diagnósticos y complicaciones concordantes con los estándares aceptados por la literatura mundial.

\section{BIBLIOGRAFÍA}

1. Bhattacharyya N, Fried MP. Assessment of the morbidity and complications of total thyroidectomy. Arch Otolaryngol Head Neck Surg 2002; 128(4): 389-92.

2. F Krause $P$, V Mercado M, J Bermeo $S$, F Tocornal J, J Vizcarra A. Estudio y tratamiento de los tumores de la glándula tiroides. Rev Otorrinolaringol Cir Cabeza Cuello 2003; 63: 9-20.

3. OERTLI D. Surgery of the thyroid and parathyroid glands. Chapter 7: Technique of Thyroidectomy. Pag 81 -87 Edit. Springer 2007.

4. Adams M, Doherty G. Conventional thyroidectomy. Operative Techniques in Otolaryngology 2009; 20: 2-6.

5. Gough I, WiLkinson D. Total thyroidectomy for management of thyroid disease. World $J$ Surg 2000; 24: 962-5.
6. Ozbas S, Kocak S. et al. Comparison of the complications of subtotal, near total and total thyroidectomy in the surgical management of the multinodular goiter. Endocrine Journal 2005; 52 (2): 199-205.

7. Efremidou E, Papageorgiou M et al. The efficacy and safety of total thyroidectomy in the management of benign thyroid disease: a review of 932 cases. Can J Surg 2009; 52(1): 39-44.

8. Shindo M, SteRn A. Total thyroidectomy with and without selective central compartament dissection. A comparision of complication rates. Arch Otolaryngol Head Neck Surg 2010; 136(6): 584-7.

9. SHEMEN L. Thyroidectomy using the harmonic scalpel: Analysis of 105 consecutive cases. Otolaryngol Head Neck Surg 2002; 127: 284-8.

10. Ecker T, Lopes Carvalho A, Jun-Ho C, et al. Hemostasis in thyroid surgery: Harmonic scalpel versus other techniques- a metanalysis. OtolaryngologyHead and Neck Surgery 2010; 143: 17-25.

11. Gac P, Cabane P, et al. Tiroidectomía sin ligaduras: evaluando ligasure precise. Rev Chilena de Cirugía 2008; 60(2): 127-31.

12. Pérez J, Urrutia V, et al. Uso selectivo de drenajes en tiroidectomías. Rev Chilena de Cirugía 2009; 61(6): 515-8.

13. Pérez J, Venturelli M. Complicaciones de la cirugía tiroidea. Cuad Cir 2007; 21: 84-91.

14. Roh J, Park J, Park C. Prevention of postoperative hypocalcemia with routine oral calcium and vitamin $\mathrm{D}$ supplements in patients with differentiated papillary thyroid carcinoma undergoing total thyroidectomy plus central neck dissection. Cancer 2009; 115: 251-8.

15. Gac P, Cabané $P$, et al. Incidencia de hipocalcemia postiroidectomía total. Rev Méd Chile 2007; 135: 26-30.

16. Asari R, Passler C, et al. Hypoparathyroidism after total thyroidectomy. A prospective study. Arch Surg 2008; 143(2): 132-7.

17. Shindo M, ChHeda N. Incidence of vocal cord paralysis with and without recurrent laryngeal nerve monitoring during thyroidectomy. Arch Otolaryngol Head Neck Surg 2007; 133: 481-5.

18. De Pedro I, Vartarian J, et al. Vocal fold inmobility after thyroidectomy with intraoperative recurrent laryngeal nerve monitoring. Sao Paulo Med J 2007; 125(3): 186-90. 